\title{
LE RENDEZ-VOUS DE VIENNE
}

Tous les quatre ans depuis 1966, l'Association internationale de radioprotection (IRPA) à laquelle sont affiliées quelque trente cinq sociétés nationales de radioprotection, rassemble lors de son congrès international plusieurs milliers de spécialistes venus du monde entier.

Cette année, le $9^{e}$ congrès IRPA aura lieu à Vienne du 14 au 19 avril, au coeur même du palais de Hofburg, sous l'emblème d'un cavalier de l'école espagnole d'équitation, maîtrisant son cheval blanc.

Le cérémonial est bien rodé : discours inaugural, attribution du "prix Sievert" à un grand nom de la radioprotection, communications invitées des présidents des organisations internationales, cours, séminaires, séances posters, exposition de matériels, ..., annonce de la prochaine ville qui recevra en l'an 2000 IRPA 10, discours de clôture.

Mais cette année est particulière.

IRPA 9 coïncide avec l'anniversaire de la catastrophe de Tchernobyl qui, il y a dix ans, a secoué les certitudes des experts et la confiance du public. Et l'on verra côte à côte les Sociétés de l'Est et de l'Ouest qui n'ont pas toujours vu avec les mêmes yeux le même événement. Au cours d'une session spéciale, le ministre allemand de l'environnement qui aura présidé la semaine précédant IRPA 9 la grande conférence organisée par les Agences internationales sur Tchernobyl, présentera le bilan qui peut être tiré de la catastrophe " 10 ans après".

Dans un tel cadre et avec une telle actualité, la Société française de radioprotection (SFRP) a voulu marquer sa présence à la fois collectivement en tant que Société et individuellement par le nombre et la qualité des présentations de ses adhérents. Pour la première fois la SFRP tiendra un stand et diffusera une brochure décrivant ses activités, son organisation ainsi que celles de sociétés avec lesquelles elle a noué des liens étroits comme l'ATSR (Association pour les techniques et les sciences de radioprotection) ou avec lesquelles un accord est sur le point d'être conclu comme la SFPH (Société française des physiciens d'hôpital). La politique d'incitation à la présentation de communica- 
tions réalisées auprès des spécialistes français a porté ses fruits puisqu'avec près de 90 posters et communications, la SFRP se situe en tête des participants. Les services offerts aux intervenants français par la SFRP devraient en outre assurer la qualité de la réalisation pratique des posters. Enfin, pour marquer sa solidarité avec les pays de l'Est, la SFRP qui a signé en 1993 un accord avec la Société tchèque de radioprotection, a financé la participation de cinq experts au congrès IRPA 9.

Cette politique volontariste de la SFRP n'a été possible que grâce au soutien financier apporté par EDF, l'ANDRA, l'IPSN, la COGEMA, le CEA, notamment le Centre de Fontenay-aux-Roses, et FRAMATOME.

Par ces différentes initiatives la nouvelle équipe de la SFRP confirme les choix d'ouverture nationale et internationale faits par les précédents présidents Laurent Stricker et Jean-Marc Cosset.

Annie SUGIER Présidente de la SFRP 